\title{
Biological denitrification of fertiliser wastewater at high chloride concentration
}

\author{
Ahmed S Ucisik* and Mogens Henze \\ Environment \& Resources DTU, Technical University of Denmark, Bygningstorvet, Building 115, DK-2800 Kgs. Lyngby, Denmark
}

\begin{abstract}
Wastewater from the fertiliser industry is characterised by high chloride concentration, normally varying between 60 and $76 \mathrm{~g} /$ $\ell$. Experiments with biological denitrification were performed in laboratory-scale 'fill and draw' reactors with synthetic fertiliser wastewater, with chloride concentrations up to $96.7 \mathrm{~g} \mathrm{Cl} / \ell$ at $37^{\circ} \mathrm{C}$; the $\mathrm{pH}$ was controlled in the range 6.8 to 7.2 . Potassium acetate was added as carbon source for the suspended cultures. The results of the experiments showed that biological denitrification was feasible at the extreme environmental conditions prevailing in undiluted fertiliser wastewater. However, the extreme conditions affected the denitrification rates. The volumetric rates were reduced by a factor of ten between chloride levels of 4.8 to $96.7 \mathrm{~g} / \ell$. The results from the performed tests can also be applied to other wastewaters with similar characteristics as that of the fertiliser industry, i.e. with high salinity. As an example, the pharmaceutical and aquacultural industries can be mentioned.
\end{abstract}

Keywords: biological denitrification; fertiliser wastewater; inhibition; high chloride concentration; high salinity.

\section{Introduction}

The fertiliser industry produces wastewater with an elevated level of salinity mainly due to the calcium chloride content. Depending on the location of the fertiliser treatment plant, the chloride concentration varies between 60 and $76 \mathrm{~g} / \ell$. In addition to this significant chloride concentration, the high concentration of nitrate-nitrogen is also an important pollutant in the wastewater.

The traditional treatment of wastewater originating from the fertiliser industry is the dilution method. The wastewater is diluted either by other industrial wastewater or municipal wastewater before being treated in wastewater treatment plants. However, the brine, such as calcium chloride in the wastewater is wasted. To enable commercial re-use of the calcium chloride, the possibility of removing the nitrate in the wastewater is investigated. Moreover, if nitrate is reduced, the environmental impact of brine would be significantly minimized and alternative disposal methods, such as open sea disposal could be developed, since only calcium and potassium chloride would remain.

Although direct electrochemical processes using a catalyst for the process can remove nitrate-nitrogen, the most attractive process for the removal of nitrate is biological denitrification. However, the information on this biological process under extreme conditions, such as high chloride concentration, is limited and it is believed that high salt content in wastewater significantly decreases treatment efficiency of conventional activated sludge, attached growth, anaerobic, nitrification and denitrification processes (Dan et al., 2003). Few studies have considered denitrification under very high concentration and as a result there is no consensus on the effect of salinity on denitrification. Vredenbregt and his colleagues (1997) studied both biological nitrification and denitrification in high salinity wastewater by using a fluid-bed reactor, and concluded that biological denitrification was effective up to $45 \mathrm{~g} \mathrm{Cl} / \ell$ where both

* To whom all correspondence should be addressed.

前+45 4525 1460; fax:+45 4593 2850; e-mail: asu@er.dtu.dk Received 6 October 2003; accepted in revised form 19 February 2004. nitrate and nitrite were removed effectively. Kristensen and Jepsen (1991) studied biological denitrification in the presence of sodium ions at high temperatures of 40 to $50^{\circ} \mathrm{C}$ and achieved successful denitrification at $30 \mathrm{~g} / \ell \mathrm{NaCl}$. The pilot plant studies for the flue gas scrubbing liquors at $20 \mathrm{~g} \mathrm{Cl} / \ell$ reported in Dahl et al., 1997, showed satisfactory denitrification rates. The maximum denitrification rate was $3 \mathrm{mg}$ N/g VSS•h. Van der Hoek and his colleagues (1987) showed that the combined ion exchange/biological denitrification process was a feasible technique for nitrate removal from groundwater, and denitrification was possible in the presence of 10 to $30 \mathrm{~g} / \ell$ sodium chloride or sodium bicarbonate. Panswad and Anan (1999b) also achieved biological denitrification at the same salinity, $30 \mathrm{~g} / \ell \mathrm{NaCl}$, where the specific nitrate uptake rate was reduced from 2.54 to $1.82 \mathrm{mg}$ N/g MLSS•h. Finally, Glass and Silverstein (1999) managed to achieve denitrification of a wastewater containing $8.2 \mathrm{~g} / \ell \mathrm{NO}_{3}-\mathrm{N}$ with ionic strength of 3.0 (total dissolved solids: $180 \mathrm{~g} / \ell$ ) by using activated sludge in benchscale sequencing batch reactors. The synthetic wastewater in their study contained $12.23 \mathrm{~g} \mathrm{KCl} / \ell$ and $71.24 \mathrm{~g} \mathrm{NaCl} / \ell$ and the $\mathrm{pH}$ of the wastewater was 9 . A similar attempt to acclimate activated sludge to denitrify the high total dissolved solids; high-nitrate wastewater at $\mathrm{pH} 7.5$ was unsuccessful. The other studies in the literature mainly focus on COD removal at high salinity levels.

The main objective of this study, therefore, is to investigate whether biological denitrification is possible at chloride levels of 60 to $76 \mathrm{~g} / \ell$ and, if so, to evaluate the performance of the biological denitrification process.

\section{Material and methods}

To accomplish this objective, 'fill and draw' reactors were operated for ten months with increasing salinity levels in order to compare the experimental results.

The system was first operated for a month to establish steadystate conditions at a chloride concentration of $4.83 \mathrm{~g} / \ell$, after which the calcium chloride concentration in the influent was increased gradually up to $96.7 \mathrm{~g} / \ell$ (total dissolved solids: $210 \mathrm{~g} / \ell$ ) during the 


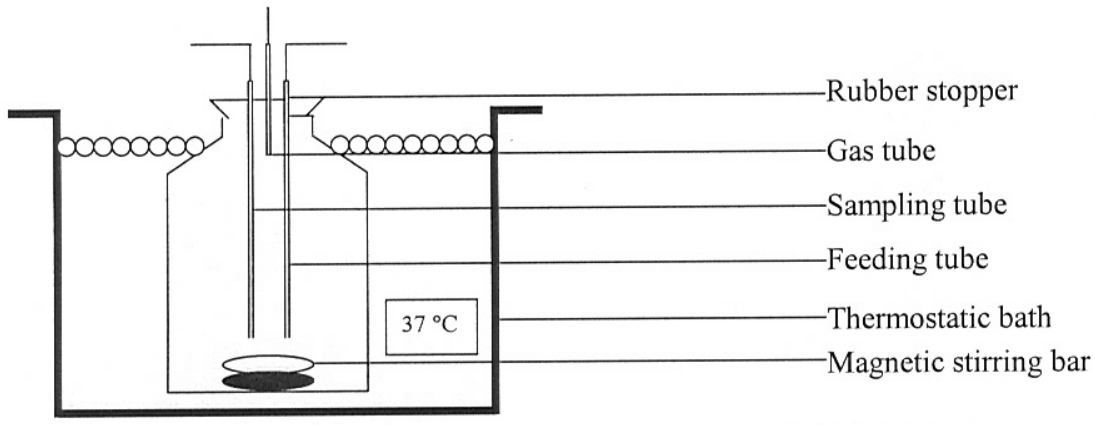

Figure 1

Experimental set-up for the biological denitrification process
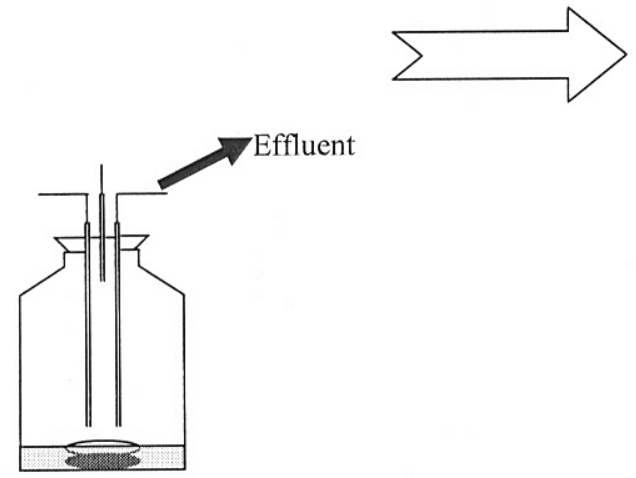

Drainage of supernatant (20 min)
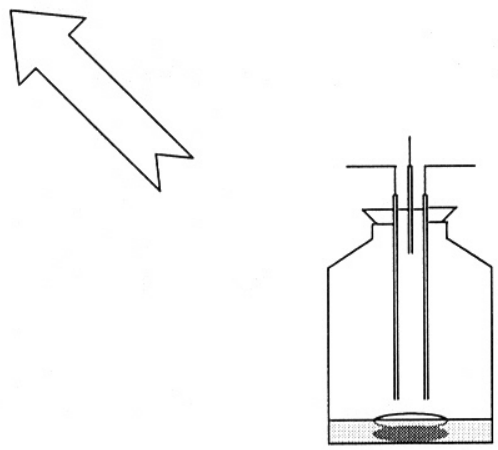

Sedimentation $(25 \mathrm{~min})$

Figure 2

Operational cycle for biological reactors

following nine months. The chloride concentration was increased gradually, since rapid shifts in salt concentration typically cause more problems (Woolard and Irvine, 1995; Kargi and Dincer, 1996).

\section{Experimental set-up}

Four 'fill and draw' reactors were operated at identical conditions in the laboratories of Environment \& Resources DTU, Technical University of Denmark. Each reactor had a volume of $5 \ell$ of which $4.4 \ell$ were used for the denitrification process. The reactors had rubber stoppers to create anoxic conditions for the denitrification process. Three tubes to provide sampling and feeding as well as to prevent overpressure in the reactor (by purging the build-up of nitrogen gas bubbles) penetrated the rubber stoppers. By placing the reactors in a thermostatic water bath the temperature was held at $37^{\circ} \mathrm{C}$ for all the reactors. Satisfactory agitation was achieved with magnetic stirring. The experimental set-up for each denitrification reactor is shown in Fig. 1.

The operational cycle is illustrated in Fig. 2. The duration of each cycle was $24 \mathrm{~h}$. One litre of synthetic wastewater was pumped to the reactors continuously throughout a period of $23.25 \mathrm{~h}$. During this period, magnetic stirrers kept the micro-organisms in the reactors in suspension. After the filling period the micro-organisms were allowed to settle for $25 \mathrm{~min}$ before the supernatant was drained off. The hydraulic retention time (HRT) of the system was regulated to $5 \mathrm{~d}$.

\section{Synthetic wastewater}

The initial wastewater was identical for all the reactors. The composition of this water for each chloride level is given in Table 1. Both potassium nitrate and potassium acetate concentrations in the feedstock were changed gradually according to the biomass activity in the reactors. When the total effluent oxidised nitrogen (sum of nitrite and nitrate) concentration measured equal or higher than $3 \mathrm{mg} \mathrm{N} / \ell$, the concentrations of nitrate and carbon sources in the feed were reduced to the initial levels for potassium nitrate and potassium acetate $-242 \mathrm{mg} / \ell$ and $918 \mathrm{mg} / \ell$, respectively (Table 1 ). After a $1 \mathrm{~d}$ feeding period, chloride concentration was increased to the next level.

Denitrification increases alkalinity. To avoid influence on reaction rates the $\mathrm{pH}$ was thus regulated in the range of 6.8 to 7.2 by adding concentrated acid solution to the synthetic wastewater, since the chosen $\mathrm{pH}$-range provided the highest denitrification rates according to the literature (Grady and Lim, 1980; Henze et al., 2001). Potassium nitrate and potassium acetate concentrations were used in the synthetic wastewater; potassium being the best antagonist for reducing the inhibitory concentration of a cation such as calcium in the wastewater (McCarty, 1964 and acetate being the best carbon source for the denitrification process (Gerber 1987; Henze, 1990; Moser-Engeler, 1998).

\section{Biomass}

The reactors were inoculated with activated sludge from the Fredericia Municipal Wastewater Treatment Plant, in which the biomass had already adapted to a chloride concentration of 4.83 $\mathrm{g} / \ell$ due to the addition of industrial wastewater from the Kemira Agro Fertilizer Plant. After a month, the steady-state conditions at the new process temperature $\left(37^{\circ} \mathrm{C}\right)$ were established and the biomass was ready for further increase in chloride concentration. The experiments were all performed at $37^{\circ} \mathrm{C}$, which was the 